\title{
Research on optimization for the mushroom-shaped root-rim structure of turbine blades
}

\author{
Qiuwan Du ${ }^{1, a}$, Jiaobin $\mathrm{Ma}^{1, \mathrm{~b}}$, Qi Jing ${ }^{1, \mathrm{c}}$, Di Zhang ${ }^{1, \mathrm{~d}^{\star}}$ \\ ${ }^{1}$ School of Energy and Power Engineering, Xi'an Jiaotong University, Xi'an, Shanxi, China, 710049 \\ aduqiuwan@stu.xjtu.edu.cn, 'bmajiaobin@stu.xjtu.edu.cn, ${ }^{c}$ jingqi@stu.xjtu.edu.cn, \\ dzhang_di@mail.xjtu.edu.cn
}

Keywords: Optimization; Mushroom-shaped; Root-rim; Pattern search; Strength performance

Abstract:The APDL (ANSYS Parametric Design Language) and mathematical optimization algorithm are applied for the optimization of the mushroom-shaped root-rim structure of turbine blades. In the optimization the better structure is obtained, and the maximum equivalent stress is notably reduced, which proves the effectiveness of the optimization method used.

\section{Introduction}

As one of the core parts in the steam turbine, turbine blade is of great importance for the safety and reliability of steam turbine. The modern architecture optimization method [1] is adopted to design root-rim structure and improve the strength properties of the blade. Several scholars such as Shankar [2] and Meguid [3] have conducted much research on the optimization of turbine blade root-rim structure. However, they usually focused on the $\mathrm{T}$ and fir-tree root-rim structures, and little attention was paid to the optimization of mushroom-shaped model, which is investigated in this paper

\section{Calculation model}

The mushroom-shaped root-rim structure of steam turbine blade is selected for optimization analysis in this paper. The calculation model with three teeth is shown in Fig.1. In the calculation a mesh size of 142969 is used. The detailed material properties of blade and rim are listed in Table.1.

The boundary conditions for calculation are shown in Fig.2, and they can be summarized as follows:1) Establishing compact pairs between root compact tooth surfaces and rim compact tooth surfaces; 2) Applying tangential and axial displacement constraints for the nodes on the rim inlet surface; 3) Applying radial displacement constraints for the nodes on the axis; 4) Applying tangential displacement constraints and coupled axial freedom for the nodes on the rim outlet surface; 5) Applying tangential displacement constraints for the nodes on the rim side faces. 6) Applying the centrifugal load with $314.159 \mathrm{rad} / \mathrm{s}$ corresponding to $3000 \mathrm{r} / \mathrm{min}$.

\section{Optimization process}

In this paper the genetic algorithm is selected for the optimization. The mushroom-shaped root-rim structure and variables are shown in Fig.3. The root-rim structure is defined with several geometric parameters, and 13 of those are selected as design variables for optimization, including the spaces between teeth $H_{1} 、 H_{3} 、 H_{5}$, the tooth thicknesses $H_{2} 、 H_{4} 、 H_{6}$, the contact surface widths of different teeth $W_{1}, W_{2}$, the rim contact surface inner radius $R_{1}$, the rim non-contact surface inner radius $R_{2}$ and the root neck widths $L_{1}, L_{2} 、 L_{3}$.

Secondly, in order to guarantee the reasonableness of modeling establishment, three state conditions are set as follows: 1) $\left.\left.H_{2^{-}}\left(R_{1}+R_{2}\right)>1.50 ; 2\right) H_{4^{-}}\left(R_{1}+R_{2}\right)>1.50 ; 3\right) G_{1}>2.00$.

Finally, the maximum equivalent stress of the root-rim structure $F_{\max }$ is selected as the objective function to be optimized. 
Table.1 Material properties

\begin{tabular}{|ccc|}
\hline Items & Blade & Rim \\
\hline Material & Steel alloy & Steel alloy \\
Density $\left[\mathbf{k g} \cdot \mathbf{m}^{-\mathbf{3}}\right.$ ] & 7810 & 7890 \\
Elastic modulus[Gpa] & 202.39 & 206.97 \\
Poisson's radio & 0.279 & 0.279 \\
Friction coefficient & 0.2 & 0.2 \\
\hline
\end{tabular}
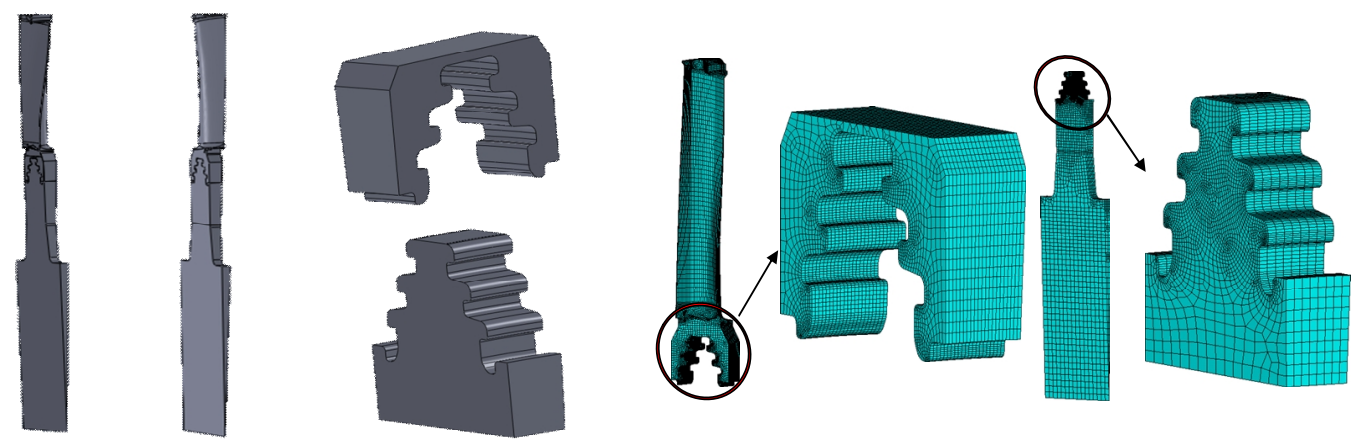

Fig.1 The caculation model
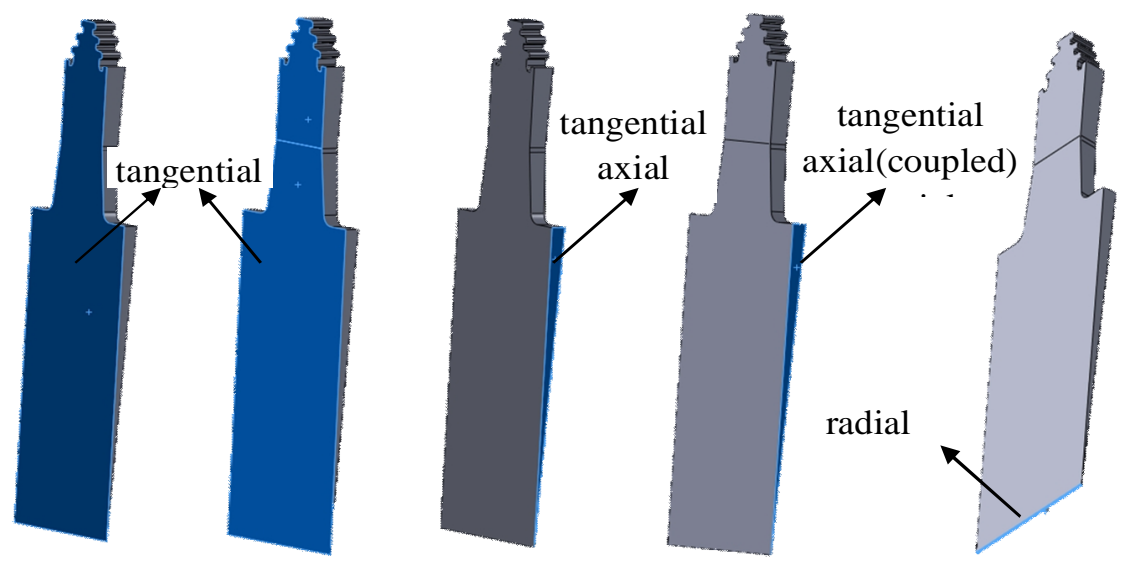

Fig.2 Boundary conditions
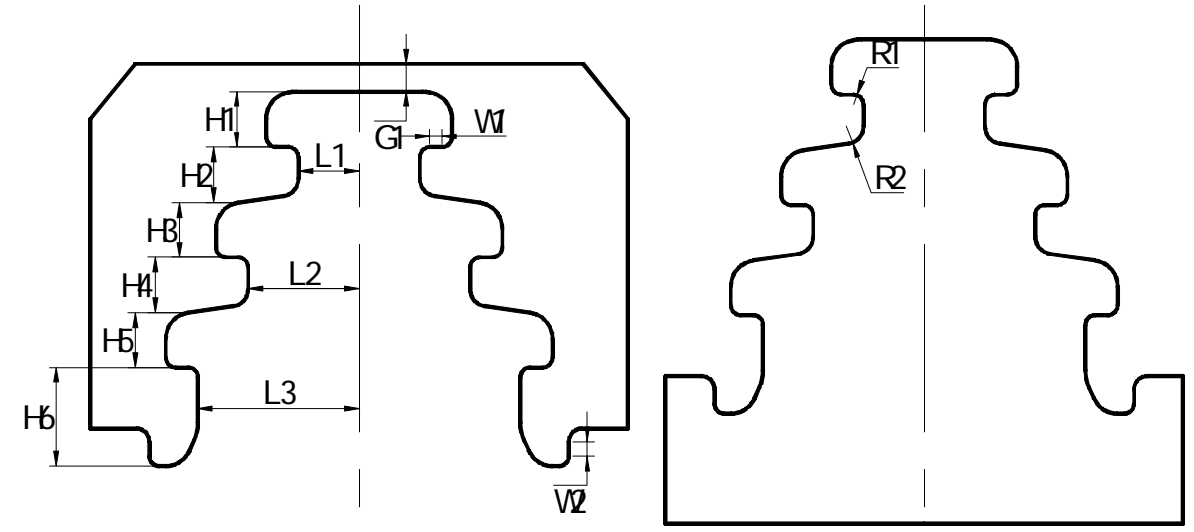

Fig.3 The root-rim structure and design variables

The changes of objective function $\left(F_{\max }\right)$, mesh size and evaluations per interval with iteration are shown in Fig.4. It can be seen that the objective function, namely the maximum equivalent stress, decreases rapidly in the initial period of optimization and after about 31 iterations, the objective function value tends to be stable gradually, slowly approaching to the optimal value $562.85 \mathrm{MPa}$. When 
the mesh size reaches to $6.1035 \times 10^{-5}$, the optimization calculations are over. The number of finite element calculations in each iteration trends to increase and stabilizes eventually in the optimization process. The overall number of finite element calculations is 502 .
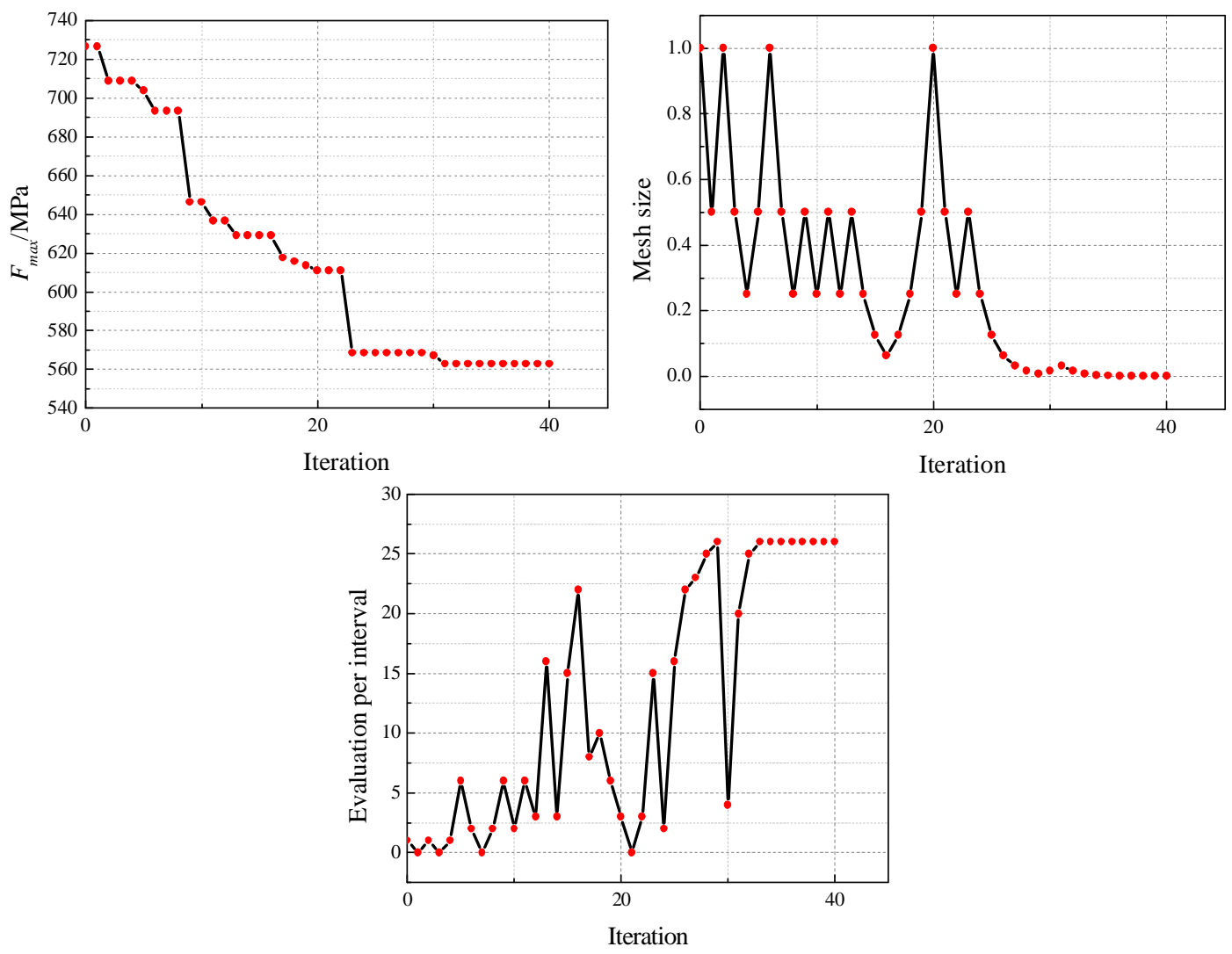

Fig.4 The optimization process

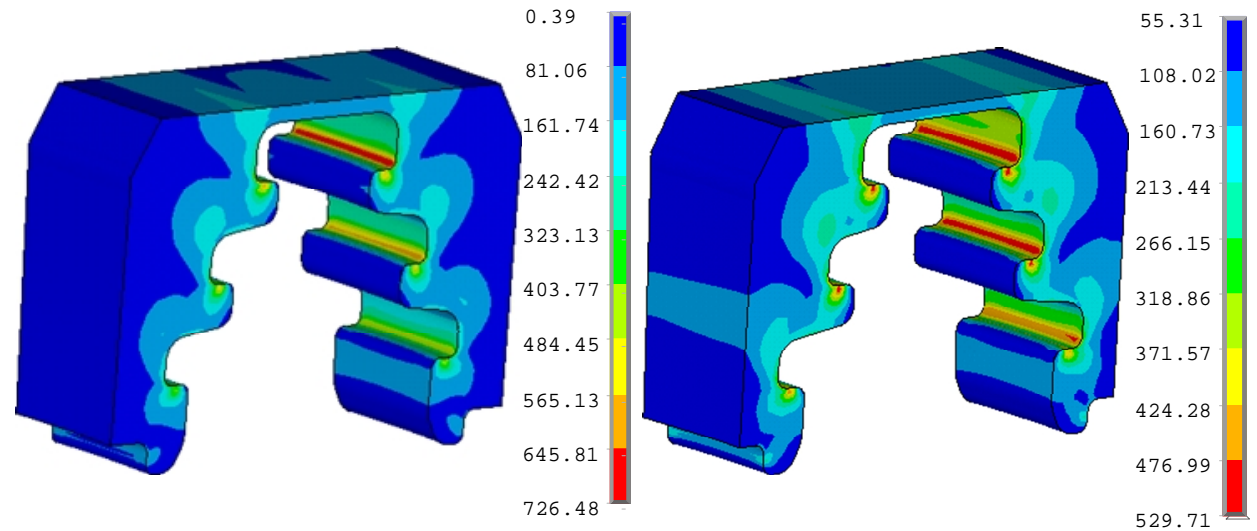

Fig.5. The von Mises stress contours of root before and after optimization/MPa.
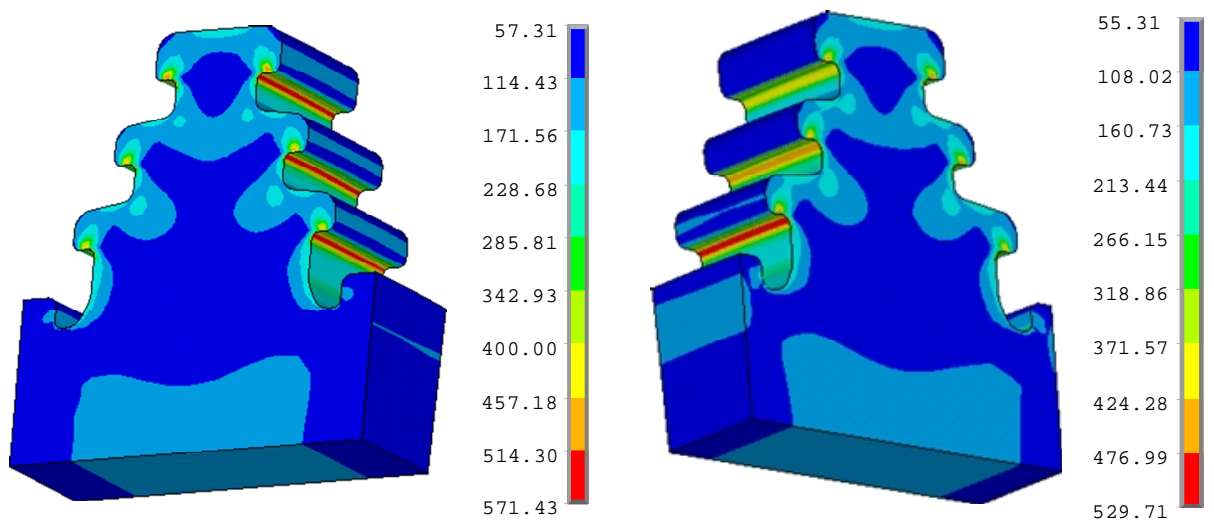

Fig.6. The von Mises stress contours of rim before and after optimization/MPa 


\section{Results and analysis}

The von Mises stress contours for the mushroom-shaped root-rim structure before and after optimization are shown in Fig.5 and Fig.6. It can be seen that the distributions of stress are basically the same before and after optimization. Besides, the stresses in the contact area and inner fillet are much larger than those in the rest areas. Through further analysis it is found that a significant decrease in the maximum equivalent stress value can be realized after optimization. The maximum equivalent stress of the root structure is $726.49 \mathrm{MPa}$ before optimization, which is located in the first fillet on the outlet side near the suction surface. While after optimization the maximum equivalent stress in the same position is only $562.85 \mathrm{MPa}$, which is reduced by $22.52 \%$. Meanwhile, the maximum equivalent stress value of the rim decreases to $529.71 \mathrm{MPa}$ from $571.43 \mathrm{MPa}$ with a notable reduction of $7.30 \%$.

\section{Conclusions}

In this paper, APDL and genetic algorithm are applied to optimize the mushroom-shaped root-rim structure. During optimization, 13 key dimensions affecting the root structure are chosen as design variables and the maximum equivalent stress of the root-rim structure is selected as the objective function. After optimization a better structure is obtained and the maximum equivalent stress is reduced by $22.52 \%$, improving the bearing condition of the root-rim structure. In the meantime, it is proved that APDL can be used for the parametric finite element analysis, and the mathematical optimization algorithm can be applied to optimize the blade root-rim structure. It is also found that the key dimensions chosen is only slightly changed, and the stress is reduced a lot. Thereby, the method is of great engineering value for the design of turbine blade structure, especially for the mushroom-shaped root-rim structure.

\section{References}

[1] Schmit LA. Structural design by systematic synthesis[C]. Proceedings of 2nd Conference on Electronic Computation, ASCE, New York, USA, October 17-20, 1960

[2] Shankar M, Kumar K, Prasad S A. T-root blades in a steam turbine rotor: A case study[J].Engineering Failure Analysis, 2010, 17(05): 1205-1212.

[3] Meguid SA, Kanth PS, Czekanski A. Finite element analysis of fir-tree region in turbine discs[J]. Finite Elem And Design, 2000, 35(4): 305-17. 